\title{
The Transformation of Archival Philosophy and Practice Through Digital Art
}

\author{
John Charles Ryan \\ Edith Cowan University
}

\begin{abstract}
In many ways, digital practices have precipitated remarkable changes in the global accessibility of art. However, the digital revolution has also radically influenced the conservation processes surrounding art, including archiving, preserving, and remembering. This paper explores the conservation of digital (or "variable media") artworks for the future benefit of culture, with particular reference to creators and viewers of art, as well as participants in interactive artworks. More specifically, this paper focuses on the philosophical and technical approaches adopted by creators, conservators, and philosophers involved in the preservation of variable media artworks. Issues of programming, interoperability between archival systems, and enhanced public access increasingly inform the design of digital archives. Indeed, the continuously shifting technological landscape — marked by the centrality of digital technologies to everyday life-problematizes the preservation of digital art through mainstream museological paradigms. Part of this analysis of digital art conservation will be drawn from the archival philosophies of Boris Groys and Rick Prelinger.
\end{abstract}

Keywords: digital art, new media, conservation, archive, philosophy

\section{Introduction}

How should we safeguard the 21 st century digital arts heritage for future generations - a heritage that remains intrinsically connected to mutable, impermanent, and fragile technologies? And how have digital practices inspired and, in many instances, necessitated new strategies for archiving and preserving art? Despite the critical role that technologies play in recording, transmission, and preservation, digital artworks are highly ephemeral and prove to be difficult to conserve through conventional approaches. Software becomes buggy and degraded, while hardware is superseded by new models and Internet sites fall into disrepair or go offline when the server space is no longer supported. In light of these challenges, this paper emphasizes the impact of digital arts practices on traditional approaches to preserving art, including phases of acquisition, collection, and archiving. Mark Tribe of Rhizome.org describes four methods of digital arts preservation: documentation (including screen grabs and artist statements), migration (applying recent technologies, such as new file formats, to artworks), emulation (using software to allow works to function on new platforms), and recreation (remaking the artwork for new technologies) (Wands 2006, 206). This paper foregrounds innovative models of archiving that have pioneered these methods - most notably the Variable Media Questionnaire (VMQ) - to encompass the changing relationships among conservators, creators, technicians, participants, audiences, and technologies.

John Charles Ryan, postdoctoral research fellow, School of Communications and Arts, Edith Cowan University, Australia; main research field: Digital Art. Email: john.ryan@ecu.edu.au. 
This paper outlines the array of conceptual and technical challenges that conservators encounter when working with digital productions. One of the primary archival considerations is the complexity of interactive, participatory and process-based works, which generally resist an object-centered approach to conservation and tend to involve multimedia components and distributed authorship. The contingencies of fragile and obsolete media — or what new media theorists refer to as "technological obsolescence" (Ippolito 2003a, 47)—require forward-looking solutions to digital arts preservation. The paper also discusses the importance of metadata in archival systems - as well as the broader correlation between preservation and technological infrastructures - in terms of promoting public access to content over networks. The preservation of digital art has transformed the role of libraries, museums, and galleries from that of collection and curation to that of co-production and co-creation ensuring the long-term viability of works through the active management of change over time. In sum, collaborative interaction among conservators, creators, and philosophers will continue to transform and democratize digital arts preservation.

\section{The Idea of Liveness: Preservation as Theory and Contingency}

This section considers the place of "the archive" in the production of cultural meaning and values, as well as the particular conservation realities faced by digital arts conservators. German-Russian philosopher Boris Groys' notion of the "cultural economy" provides a way of thinking about the fundamental place of the archive in culture. Digital theorist Rick Prelinger characterizes the "accessible archive" as necessitating a community of users, whereas conservation theorist Vivian van Saaze advocates the principles of change, intervention, and production in archive management. Moreover, German media theorist Wolfgang Ernst theorizes digital memory as "working memory" that crosses between the hardware-based memory of computers and the cultural memory of human users. Echoing van Saaze's principles, Josephine Bosma identifies technological obsolescence and the decline of audience participation as some of the multiple factors that jeopardize the preservation of variable media productions. Furthermore, media theorist Philip Auslander conceptualizes digital arts preservation through the notion of performativity in which highly ephemeral creations must be re-performed regularly to retain their value, meaning, and impact. In these terms, performativity becomes an essential component of digital arts conservation plans. A case study of Mark Napier's net.flag (2002) also explores ideas of crowdsourcing, tacit knowledge, and acceptable loss in relation to digital arts preservation.

In describing the role of the archive broadly in Western history, culture, and society, Boris Groys proposes the term "cultural economy" as "the exchange that takes place between the archive of cultural values and the profane space outside this archive" (Groys 2012, 1). By definition, an archive preserves immaterial (digital) or material (artefactual) items of cultural importance. Conversely, it excludes irrelevant or invaluable things, relegating these items to the "profane" world that exists outside of the archive's ambit. Most importantly, the boundaries of the cultural archive are fluid and changing. Things in the profane space become valued and those in the archive become irrelevant or ignored over time (Groys 2012, 1-2). For Groys, the determination of what is significant and, therefore, to be included in an archive (what he terms the "New") and what is irrelevant and, therefore, to be excluded (what he calls the "Old" or "non-collected reality") is a matter of power and politics. More than a representational system or mechanical structure, the archive actively furnishes the groundwork for the future manifestation of historical, cultural, and artistic forms:

The function of the archive cannot consist merely of illustrating or representing history or of holding fast to the memories of history the way in which this history took place "in reality." Rather, the archive constitutes the prerequisite for 
something like history to emerge in the first place, because only if the archive is already, there are we able to compare the New with the Old... The archive is a machine for the production of memories, a machine that fabricates history out of the material of non-collected reality. (Groys 2012,3)

For Groys, an archive operates according to an internal logic and coherence, attracting material that is integral firstly to itself and not to society or culture per se. In its capacity to establish and preserve the "New," the archive is an arbiter of cultural value and an actant in the course of history. In short, the flow of content in and out of an archive does not only mirror shifting societal values (i.e., an item in the profane world is judged of value and included in the archive). Rather, the multi-dimensional movement between the profane and the archive dynamically underpins the cultural economy's existence. In Groys' terms, the archive is a living repository (although he prefers the term "machine") for future creative production; by inference, the archival space is something like the sacred.

Like Groys, Rick Prelinger theorizes the act of archiving as a crucial mode of cultural production. For Prelinger, an "accessible archive" is one in which preservation and access are not static concepts. An archive should engage a network of users, creators, and conservators. Mirroring Thomas Vander Wal's concept of the folksonomy, Prelinger advocates the work of citizen archivists and community members. For both Groys and Prelinger, archival work is that of "cultural producer," facilitating the production of new works from archival materials without inducing unnecessary anxiety over copyright and other regulations that stifle creativity (Prelinger 2009, 173). A "sandbox" model of the archive would make available a testing environment in which artists could experiment with archival material. As such, Prelinger's ethos of the archive is comparable to Lawrence Lessig's articulation of "free culture," in which innovation, creativity, and risk are nurtured rather than quashed by legal structures. As a "new folk art," archiving is "widely practiced and has unconsciously become integrated into a great many people's lives, potentially transforming a necessity into a work of art" (Goldsmith, 2011a on the work of Prelinger).

In the context of open access to archival information, the Internet Archive (IA) (www.archive.org) is a massive digital repository founded in 1996 by American computer scientist Brewster Kahle. In addition to preserving texts, audio, video, and software, the IA includes a service called Wayback Machine that archives websites and grants users access to previous web content as it appeared originally. As of 2012, the IA contained 10 petabytes of data with the Wayback Machine archives accounting for 2 petabytes or $20 \%$ of the total. (A petabyte is equivalent to one quadrillion bytes of data) (Claypoole \& Payton 2012, 9). The IA also holds about 5,000 digital files of films and footage from the Prelinger Archives, founded in 1983 as a traditional and site-based archive in New York City (Internet Archive, 2013).

Another key precept in the literature of digital arts preservation is digital memory. Put in broad terms, the practice of digital memory involves cultural heritage preservation through digital approaches. Wolfgang Ernst theorizes the interface of memory and new media. In the digital era, cultural memory is intimately connected to the technical memory of computers as part of an active and integrated process Ernst calls "working memory" (Parikka \& Ernst 2013). However, the problem is that, while memory can be individual, collective, or institutional, the notion of digital memory is often conflated with data storage. For Ernst, digital memory is a dynamic form of cultural memory that emerges through human use of social media and participation in “everyday media environments" (Parikka \& Ernst 2013, 16). Digital memory comprises the storage capacities of devices, the structure of archives, and the faculty of human memory - both individual and collective modes. Without a doubt, the boundary between the storage of data (technical, digital) and the practice of operating the 
storage device (cultural, habitual) has become ever-more indistinct. In a similar sense, Bosma $(2011,177)$ describes "the archive of the real" as the amalgamation of archival technologies, new media sources, and oral memories transmitted through the Internet. As the preservation of cultural memory, the archiving of digital artworks requires traditional materials (e.g., documents) in conjunction with multimedia (e.g., audio and video) (Bosma 2011, 188). The cultural memory work of media scholars like Wolfgang Ernst and Josephine Bosma protects against the "digital dark ages" in which data loss has multiple and negative implications for technology and society (Bosma 2011, 185).

In order to appreciate the contingencies negotiated by digital archives, it is important to have a sense for traditional approaches to arts preservation. On the whole, mainstream strategies strive to keep intact the original state of an object of art through the control of its environment and the exclusion of certain influences, most prominently human interaction (see, for example, Richmond 2012). An object is typically protected from deleterious external variables - including light, heat, vibration, and physical wear from handling by people. However, museological practices and basic definitions of what constitutes an archive have been transformed and expanded by the special demands of digital preservation. As Bosma points out, digital artworks require that archives evolve beyond "specific locations (museum archives) and the on-site conservators" (Bosma 2011, 179). Moreover, digital artworks often create meaning only in reference to a technologically-based environment and a community of users participating actively in the production of the work over time (for example, see Mark Napier's net.flag). As a consequence, software obsolescence, diminished technological support or failure to engage an audience, might compromise the long-term evolution of digital artworks (Bosma 2011, 165). Hence, conservators must become intimately acquainted with the design and ethos of ephemeral works to ensure their longevity: "To preserve art that is produced using unstable media, or to restage any process or time-based works, it is necessary to know the artist's intent and work process" (Bosma 2011, 171).

Mark Napier is a software developer and one of the pioneers of net.art. His project net.flag (2002) is exemplary of early Internet art, particularly for the work's interactive and participatory aspects. Acquired by the Guggenheim Museum for their permanent collection, net.flag (2002) confronts themes of national identity and nationalistic allegiance (see http://webart.guggenheim.org/netflag/). The Guggenheim is acquired net.art (consisting of the software and the continuously changing content) on the condition that the work "always be on view" (Napier quoted in Dietz 2005, 88). Napier's request reflects a simple yet profound inversion of the norms of mainstream acquisition where most art objects (paintings, photographs, sculptures, or installation pieces) could not be made publicly available at all times. In its ever-shifting content, Net.flag expresses how the Internet has radically transformed human identity by dissolving the physical borders between nations. The profusion of interactive platforms - more recently manifested as social media - ensures that content is accessible on a round-the-clock basis. In Napier's work, the flag as a relatively stable symbol of nationhood is supplanted by the flag as a constantly changing form—one evolving constantly and democratically:

Net.flag explores the flag as an emblem of territorial identity by appropriating the visual language of international flags. An online software interface makes this language of shapes and colors available to anyone with web access. The visitor to net.flag not only views the flag but can change it in a moment to reflect their own nationalist, political, apolitical, or territorial agenda. The resulting flag is both an emblem and a micro territory in its own right; a place for confrontation, assertion, communication, and play. (Napier, n.d.)

Some media art commentators acknowledge the artwork as "an emblem for the Internet as a new territory, one composed by people from various geographical regions and ideologies" (Ippolito 2003b, 109). 
Fulfilling Napier's initial request, the Guggenheim archive of the sole copy of net.flag makes the work available to anyone at any time over the Internet. An unregistered visitor to the website can devise a new Internet flag in real time by manipulating preset motifs, including stars, colour fields, and shapes. Net.flag employs Java programming to enable users to construct a flag as the flag of the Internet (until another user decides to modify it). For Napier, the Java applet programming and the computer operating system only support the existence of the artwork, which is, instead, the algorithm built on the infrastructure making possible a high standard of user interactivity. The recognizable symbols of countries meld within each new flag, subverting the primacy of any single nation's identity. Users select the "change the net.flag" option from the right side of the webpage. There are also columns for "anatomy of the flag" and "flags of the web." A "statistics" navigation bar provides a summary of the "elements" appearing in the Internet flags. For example, the red stripes of the United States flag appear 6,226 times and the cross of the Greek flag shows up 5,366 times in different flags. The countries with the highest frequencies of their visual motifs across all flags include the United States (7\%), Australia (5\%), Greece (5\%), Argentina (2\%), and Ghana (1\%). The top-ranking elements include unity, valour, purity, peace, and harmony. The work also includes a "browse history," summarizing the overall symbolic dimensions of the current flag. In terms of preservation, net.art exhibits all four behaviours of the Variable Media Questionnaire explored later in this paper. It is interactive, duplicated, encoded, and networked and, therefore, one of the earliest and most important examples of the archiving of Internet art by a major institution.

The net.flag case study suggests the kinds of negotiations that transpire between a creator (Napier) and the archiving organization (the Guggenheim). Web-based works, such as Napier's Internet flag, encode preservation principles inherently in their design and delivery, but such works do require regular maintenance and creative infusion. Moreover, practical concerns, particularly web hosting and website obsolescence, impact the long-term success of preservation strategies. On a related note, Boris Groys' essay "The Restoration of Decay" addresses the importance of dialogue between conservators and artists during the preservation process. He characterizes the conservator as a co-creator, interpreter, conductor, director, or active interpreter of a digital artwork (Bosma 2011, 169-70). For example, works of browser or code art require that the conservator makes crucial decisions about their reconstruction or re-enactment. The preservation of code necessitates that the conservator becomes, to an extent, a creator who re-enacts the code. While some conservation strategies draw from analogue approaches to minimize impacts, other strategies use open archival formats to maintain public contribution to the content, structure, and meaning of a digital work (Bosma 2011, 166). With prescience, artists, such as etoy, embed a preservation mechanism in the design of works through public domain or open-source approaches, community-based contribution, and other techniques that promote interactivity (Bosma 2011, 175). An international collective founded in Zurich in 1990, etoy consists of media theorists, coders, architects, and engineers (Petrović-Šteger 2011, 146). Their long-term project Mission Eternity (2005) highlights issues of cultural memory, memorializes the death of loved ones, and rethinks the notion of the afterlife through the digital traces left by participants facing death. The ethos of the project is that people leave behind their physical selves as well as voluminous amounts of information about their lives - both of which constitute how the living remember the dead (Petrović-Šteger 2011, 147). A "multi-modal post-mortem activity plan" includes the Arcanum Capsule, an interactive "digital portrait" of subjects who have passed on (Petrović-Šteger 2011, 147). The portrait preserves the digital signatures of participants, including photos, voice recordings, biodata, and social network mappings. 
Performativity is another key principle for understanding the preservation of digital artworks. Artworks face obsolescence and death; like people in ill health, their ability to perform declines and must be restored or reinvigorated to exist in the future. For the etoy collective, human death, like art, is a kind of performance recorded and re-enacted over a body of dispersed materials, even after the mortal body has perished. Similarly, recordings must be reactivated or re-performed to be experienced in the present, that is, to avoid obsolescence. As a consequence of its very nature, digital art is performative in two senses. Firstly, its forms and styles on the whole must be experienced interactively in real time. Secondly, many classic works of digital art have become extremely difficult to access or re-create and, accordingly, must be re-performed as part of a conservation plan. As we have seen through a number of other works, digital art profoundly redefines notions of documentation, conservation, and the archive in terms of performativity. For example, as Philip Auslander and others have observed, it is ironic that most people come to know the first generation performance artists of the 1960s and 1970s through photographic documentation of their performances. Auslander argues that the idea of "liveness" is itself dependent on the possibility that a performance can be recorded in some way (Auslander 2006). In sum, documentation and archiving are performative in that they reflect "an artist's aesthetic project or sensibility... for which we are the present audience" (Auslander 2006, 9). In this respect, the Variable Media Network model designates "performance" as a conservation category "whenever the re-creators have to re-enact original instructions in a new context" (Ippolito 2003a, 48-49).

In his essay "Learning from Mario" (2010), Jon Ippolito considers the "crowdsourced" model of preservation - common in gaming environments - as an alternative to the centralized approaches of mainstream preservation. Crowdsourcing is a significant feature of participatory culture in the digital era. It is a problem-solving approach that enables an institution to acquire content, services, or concepts through the efforts of a large group, community, or "crowd." However, in contrast to commons-based online projects, such as Wikipedia, that offer users a shared platform to work collaboratively, crowdsourcing is initiated and managed from beginning to end by the company, organization, organizers, or other initiating institution (Brabham 2013b, 120-1). Coined in 2006 by Jeff Howe of Wired Magazine, crowdsourcing:

represents the act of a company or institution taking a function once performed by employees and outsourcing it to an undefined (and generally large) network of people in the form of an open call... The crucial prerequisite is the use of the open call format and the large network of potential laborers. (Howe cited in Brabham, 2013b, 120)

In crowdsourcing, creative production occurs at the interface between the public (the crowd) and the managing party (the institution). This combination of "bottom-up, open creation by the crowd" and top-down management by the organization characterizes crowdsourcing as a creative, although not entirely collaborative, approach (Brabham 2013a, XXI). In the context of digital art preservation, crowdsourcing draws from the skills of amateur archivists - thus calling into question the role of centralized repositories having limited public participation and access. As an exemplary initiative, the Variable Media Questionnaire (VMQ) captures quantitative and qualitative information about digital artworks, including recorded interviews with creators, programmers, and artists. Whereas the first two versions of VMQ are stand-alones, the most recent version is Internet-based, allowing the public to register their opinions about the work, and, thereby, drawing from the crowdsourcing approach to digital art preservation (Ippolito 2010).

In outlining the challenges of digital preservation, Ippolito features the example of "The Erl King" (1982-85), a collaboration between experimental filmmaker Grahame Weinbren and Roberta Friedman. Using 
multimedia features that invited participants to interrupt the production at any time, "The Erl King" was one of the first works of interactive video art or "narrative cinema" of the 1980s. It was based on two major texts: Johann Wolfgang von Goethe's poem "Der Erlkönig" (1782) and a dream analyzed by Austrian psychoanalyst Sigmund Freud (1856-1939). The technological components of the project included PASCAL code that integrated information from a SMC-70 computer, a custom built video switcher and three laser disc players. The PASCAL controlled the loading of graphics, text, video, and audio files. In 2003, Ippolito and Guggenheim curator John Hanhardt proposed "The Erl King" as a pilot study of media art preservation using the Variable Media Network (VMN) model. The work's hardware was 20 years old at the time. As a result, the conservators chose "emulation" in which a newer computer impersonated the original, allowing the execution of the source code and reproduction of the intended user experience, including the periods of lag or delay during which different components communicated. In general, emulation entails the replacement of outmoded or multi-component hardware through programming. Weinbren and Friedman worked with a VMN software engineer to develop a new platform for the code on a single computer using Java programming language. The platform mimiced the video switcher, the graphics cache, and laser disc players to guarantee the correct timing and sequencing of the work as set out by the artists. The emulation process required programming in delay, such as disc search time, to imitate the user experience of the original work based on new technologies of the 1980s (Weinbren, n.d.).

"The Erl King" is evidence of how digital arts preservation requires a variety of living, dead, and mechanical actants. For Vivian van Saaze, "continuous intervention" should be the core principle pursued by museums and other conservators to preserve new media works, those that defy the notion of art as material objects only. Following the actor-network theory (ANT) of French philosopher Bruno Latour and others, van Saaze identifies a "collective of actants" involved in the conservation of ephemeral works. Digital art conservation requires the dynamic interplay of actants: "materials, humans, spaces, spatial arrangements, procedures, and protocols" (Van Saaze 2009, 21). Human and non-human actants also include the building, its administration, technicians, curator, conservator, director, and artist. The acceptance of notions of "change, intervention, and production" in arts conservation contrasts starkly to the "hands-off or minimalist intervention approach of traditional conservation ethics" (Van Saaze 2009, 21). In order for conservation practices to accommodate digital works, change should be linked to productivity and potentiality rather than loss or damage. Indeed, traditional conservation discourses assume the fixed identity of the artwork - as an entity that must be controlled physically and conceptually in order for conservation to succeed. However, the restoration of a work back to its original state does not need to be the guiding ethic in all cases; works such as Napier's net.flag exist only in relation to change. The context of a digital artwork is complex and consists of "tacit knowledge" - sensory and conceptual information, including artistic purpose, sociocultural meanings, and the practices involved in re-creating the work (MacDonald 2009, 61).

The final, core theoretical and ethical issue surrounding the preservation of digital artworks addressed in this paper is "acceptable loss" (Harvey 2012, 76). As the literature of digital heritage shows, what is considered acceptable loss varies considerably and depends on creators, conservators, technicians, and users. Theorists of digital archiving agree that loss of content and functionality is unavoidable and that acceptable loss should generally be factored into conservation strategies. However, a 2003 joint report on digital archiving and preservation by the U.S. National Science Foundation and the E.U.-based DELOS Network of Excellence on Digital Libraries asks: "How can we measure what loss is acceptable? What tools can be developed to inform 
future users about the relationship between the original digital entity (artwork) and what they (users, viewers, participants) receive in response to a query" (Harvey 2012, 76). Moreover, according to the UNESCO Guidelines for the Preservation of Digital Heritage (2003), there are two major axioms of digital preservation, the first of which definitively rules out access as acceptable loss: "digital materials cannot be said to be preserved if access is lost" and "digital preservation must address threats to all layers of the digital object—physical, logical, conceptual, and essential” (UNESCO 2003, 21).

Digital art further redefines traditional conservation paradigms by accepting transformation, degradation, and, in some instances, loss. Regarded as negative attributes of conservation in the context of the most traditional and object-oriented artworks (paintings, sculptures, furniture), transformation and degradation are often acknowledged by digital artists or even incorporated into digital works (Bosma 2011, 171). However, excessive loss can permanently alter the original vision of the creator. Areas of acceptable loss can include the physical deterioration of the work itself, the decline of the media used to record the work, the obsolescence of technologies originally employed to execute or access the artwork, or the loss of information that contextualizes the intentions of the artist (Bosma 2011, 171). To avoid the distortion of the project and ensure an ethical conservation plan, acceptable loss needs to be delineated clearly in relation to digital artworks because many of these works rely on original conceptual aims (Stringari 2003). In particular, loss can occur when restoration or re-creation is based solely on what is documented about the work, to the exclusion of unknown, undocumented, or disregarded tacit knowledge about the work. As with digital arts conservation generally, the management of acceptable loss is the most optimal in dialogue with the artist and conservators, helping to safeguard the conservation process from misinterpretation and the "loss" elements of the work from misidentification. Indeed, one of the goals of the Variable Media Network (VMN) is to identify the acceptable range of loss through documented interactions among artists, technicians, audience members, and conservators.

\section{Scoring the Work: Digital Arts Preservation Through Notation Systems}

The previous section outlined the principal theoretical issues affecting the preservation of digital artworks. This section examines key initiatives in the history of digital and media arts preservation, most notably the Capturing Unstable Media Conceptual Model (CMCM) (2003), the Documentation and Conservation of the Media Arts Heritage (DOCAM) (2005), the Media Arts Notation System (MANS) (2007), and the Performance Art Documentation Structure (PADS) (2008). The purpose of this section is to provide an overview of the history of notation systems in the preservation of digital artworks. Appearing after the year 2000, these four systems operate according to notation systems that generate a "score" of the work through the use of descriptive metadata. Curator Richard Rinehart (2004b, 3) defines a score as "the clearest type of description that compiles formalized (systematic) discrete elements into documents that aid in the re-performance or re-creation of works of art." The section includes a case study on the Variable Media Network (VMN), featured because its principles form the basis of other notation systems for preserving digital artworks. The section concludes with a case study featuring UbuWeb as the repository of avant-garde art and applying the concept of the "gift economy" to free online archives. The preservation of digital art through scoring systems and open-access repositories is moreover a matter of digital heritage protection necessitating the collaborative efforts of a number of parties. As Josephine Bosma $(2011,173)$ argues, the preservation of digital art demands "structural changes" within and between organizations to make possible extended collaborations with external partners in the long-term interest of artworks. 
One of the first digital arts preservation strategies was the Capturing Unstable Media Conceptual Model (CMCM) (2003), developed at the V2_Organisation, an interdisciplinary centre for art and media technology in Rotterdam, in Netherlands (Fauconnier \& Frommé 2004). The central aims of the initiative were archival interoperability and the development of an "ontology" that could be used to describe relationships between the facets of an electronic or unstable media artwork. The CMCM recognized the challenges of determining the original state of an artwork and, therefore, sought to document processes, contexts, materials, practices, collaborations, and user interactions. Archival interoperability allowed individual institutions to input data on specific artworks, assembling a broader snapshot of the perspectives of external curators, critics, and conservators. An ontology resulted from a focus on considering processes instead of artworks as fixed entities and the attendant shift from object-oriented models of art preservation to process-oriented approaches. The CMCM documentation methodology recorded various interactions among software, hardware, and networks through the creation of metadata and the use of interviews about user exchanges, user numbers, intensities of interactions among users, physical contexts, and sensory modes.

The DOCAM Research Alliance was founded in Montreal in 2005 by the Daniel Langlois Foundation for Art, Science, and Technology. DOCAM (Documentation and Conservation of the Media Arts Heritage) develops and implements models for digital arts preservation through the ethos of heritage protection. Most significantly, the DOCAM framework involves documentation throughout an artwork's lifecycle, building in crucial preservation strategies from the inception of the work. DOCAM conservators record the perspectives of contributors, generating a graphic representation of the relationships between items of documentation and producers, as well as the phases, iterations, and components of the work (Daniel Langlois Foundation, 2013). The framework builds a hierarchical description, ensuring the completeness of documents, agents, and sources, and safeguarding the distinctive nature of digital artworks through a lifecycle approach. An artwork's lifecycle spans creation, dissemination, critical reception, and custody - the latter phrase including aspects of cataloguing, curating, and conserving. The hierarchical scheme is used by music cataloguing systems (such as the Functional Requirements for Bibliographic Records or FRBR) and distinguishes among the terms "work," "expression," "manifestation," and "item." "Work" is "a distinct intellectual or artistic creation;" "expression" is "the intellectual or artistic realization of the work;" "manifestation" is "the physical embodiment of an expression of a work;" and "item" is "an exemplar of a manifestation" (Iseminger 2012, 46). The documentation process begins with artists and collaborators, then broadens to include conservators, curators, and critics. It includes the installation, preservation, and restoration of the works based on the belief that, in many cases, documentation survives the original work and its creator. Documentation comprises a range of material such as 3D animation files, acquisition reports, bibliographies, copyright agreements, critical essays, exhibition leaflets, screen captures, and technical booklets. The DOCAM framework reflects three attributes of digital artworks: (a) they demonstrate a basis in technology through the use of devices that are often developed or modified by the artist; (b) they produce a range of effects related to light, sound, movement, and other behaviours; and (c) they are variable over time, especially as we have seen with participatory Internet-based works such as Napier's net.flag.

Initially developed by curator and theorist Jon Ippolito, the Variable Media Questionnaire (VMQ) (2000) is the forerunner of the DOCAM, PADS (Performance Art Documentation Structure), and MANS (Media Arts Notation System) frameworks. The VMQ evolves out of the broader efforts of Variable Media Network (VMN), an initiative of the forging the future consortium on digital curation. More specifically, the VMQ emerges from 
the Guggenheim Museum's preservation of video and conceptual art (Wands 2006, 206-7). There are now the first, second, and third generation questionnaires, designed by Ippolito at the Guggenheim Museum (2000), curator Alain Depocas at the Langlois Foundation (2003), and programmer John Bell at the Still Water research centre at the University of Maine (USA) (2010), respectively. The VMQ allows creators and users to develop guidelines for "translating their works into new media once the original medium has expired" (Forging the Future, n.d.). Creators identify the most appropriate method for translating their works, including storage, emulation, migration or reinterpretation, or, in Bruce Wands' analogous terms, documentation, emulation, migration, and recreation (Wands 2006, 206). The VMQ requests that creators - rather than archivists, critics, or new media experts - outline the mechanical components and conceptual dimensions of a work that are essential to preserving or recreating it. The Variable Media Network requires that decisions concerning the preservation of unstable artworks be made when the work is acquired by a holder (Stringari 2003). The VMQ produces a narrative of the life of a work, relying "on the sharing of stories and individual experiences told by artists and related participants in the creation, exhibition, and collection of art" (Hanhardt 2003, 8). The narrative also serves as a "score or documentation form for unstable works of art" (Bosma 2011, 171). Notation systems, such as the VMQ, describe the work and provide the basis for its recreation or re-enactment.

Ippolito's essay "Accommodating the Unpredictable" (2003) outlines the philosophy and approach of the VMQ shortly after the second version is released. Building on the knowledge artists have about their works, "the variable-media approach asks creators to play the central role in deciding how their work should evolve over time, with archivists and technicians offering choices rather than prescribing them" (Ippolito 2003a). On a case-by-case basis, the VMQ identifies "behaviours" or "medium-independent, mutually-compatible descriptions of each artwork," documenting crucial factors such as installation space, lighting requirements, and the ratio of elements (sound, wind, visual features). In VMQ terms, "performance" refers to the re-creation or re-enactment of the work in a new setting using the work-specific protocols developed by the VMQ in conversation with the artist. "Reinterpretation" is a major strategy used in the VMQ to address the technological obsolescence of the work's hardware or software components. The VMQ uses the following categories to describe works: reproduced, duplicated, interactive, encoded, and networked.

"Reproduced" refers to the work that diminishes in quality when copied to a medium such as video and audio. Duplicated works can be replicated identically through programming, such as Java applets or event-based sequences, evident in Felix Gonzalez-Torres's Untitled (Public Opinion) (1991) in which a massive spillage of candy occupies the floor space. Napier's net.flag falls into this category and the next two. Interactive works require that the artist decides whether traces of previous participants or visitors should be erased or preserved in future incarnations of the work. Encoded artworks involve programming and are accessed through networks. Weinbren's "The Erl King," discussed in the previous section, is an example of an interactive video and networked digital creation. Furthermore, the VMQ defines the "migration" of an artwork as the upgrading of its medium to a current standard. VMQ3 allows artists to compare the perspectives on their works presented by curators, conservators, technicians, and artist assistants (see the next section for a brief discussion of the latest version). Based on the VMQ, conservators Caitlin Jones and Lizzie Muller use four processes to document media art: artist interviews, audience interviews, data structure scoring, and access points identification (Jones \& Muller 2008).

Extending the VMQ model, other digital art preservation strategies make use of notation systems for scoring. Established by American curator and digital artist Richard Rinehart, the Media Arts Notation System 
(MANS) is a conceptual framework for preserving digital and related media works (Rinehart 2007). The MANS is one of the first formal notation systems for preserving digital art. Rinehart's approach to preservation responds to the "ephemeral, documentary, technical, and multi-part nature" of digital creations, as well as their "performative," "behavior-centric," and "variable" qualities (Rinehart 2007, 181). Just as a musical composition can be "re-created" across a variety of instruments while still being recognized as the original, digital art is comparably variable. In Rinehart's system, the structure of an artwork is preserved and re-created through the use of a score (Bosma 2011, 172). The score preserves the interrelationships between the work as a whole and its technical components. The process takes into account the relationships between creators and users, instances of distributed authorship and the nuances of open-ended digital arts projects (Rinehart 2007, 185). MANS notation captures enough detail to result in a record of the artwork in conjunction with relevant descriptive metadata. Moreover, the model implements tags or what are referred to as "behaviours," are developed by the Variable Media Network. These include works that are "contained" (no additional infrastructure required), "installed" (specific to spaces, sites, or placements), "performed" (process-oriented); "reproduced" (involving a loss of quality when recorded), "duplicated" (in which the copy and the original are indistinguishable), "encoded" (requiring the interpretation of computer code), and "networked" (present in more than one physical location) (Rinehart 2004a, 2-3).

Developed by curator Paul Clarke, the Performance Art Documentation Structure (PADS) is a similar notation system designed to record the components of a performance work-including, for example, audiovisual material, interviews, and objects (Gray 2008). The PADS model presents a notation system of value to performance art researchers and which characterizes the relationships between components of a work. In addition to capturing technical information, PADS records the parties involved in identifying an artwork's constituent parts - creators, users, curators, conservators, or audience members. The process foregrounds the importance of interviews with artists, curators, technicians, audience members, and archive users in formulating a score. The PADS draws extensively from the Variable Media Network model, while addressing the specific challenges of performance art preservation. Like the VMN and Rinehart's MANS, the PADS model avoids categorizing works by media type (for example, painting or sculpture) and instead applies behavioural tags, such as those mentioned above. An "interoperable" system results from the use of common metadata usage. The MPEG-21 metadata framework (explored technically in the next section of this paper) allows the distribution of performance art scores between archives.

The first work selected to receive a PADS scoring is "I Never Done Enough Weird Stuff" (1996) by British performance, installation, and video artist Richard Layzell. The scoring begins with a series of interviews with Layzell from the perspectives of curator (focused on context and influences) and conservator (highlighting the specifics of the work). The process identifies the key elements as objects, garments, roles, space, location, audiovisuals, electronics, and gestures. For example, the score, as it appears on the STARS (Semantic Tools for Screen Arts Research) archive, lists "torch" as one of the component objects. An interview with the artist reveals the provenance of the object data: "Richard's torch was a small, black Maglite, a novel item at the time as the brand was largely unknown in the UK. It was used in several performances as an "antidote" to theatrical lighting... It is hidden in Richard's inside pocket for much of the performance" (STARS, 2013).

Founded in 1996 by American poet Kenneth Goldsmith (b. 1961), UbuWeb (www.ubuweb.com) is a volunteer-based and curated collection of "avant-garde" artworks, including sound art, visual works, film, and 
poetry. Particularly focusing on obscure, out-of-print, and small-run works, the archive is replete with "the detritus and ephemera of great artists" (Goldsmith 2011b). Hosting over 7,500 artists and several thousand works, the digital archive has none of the embellishments of most websites - even non-commercial ones. The interface is "flat" and "cool," avoiding any "illusionistic depth-of-space" (Goldsmith 2002, 2). UbuWeb uses no advertisement banners, institutional logos, or donation-soliciting icons; its server space and bandwidth are donated by universities, as Goldsmith points out. UbuWeb is known for uploading sound and video files without seeking the permission of authors in an inversion of copyright protocol. This maverick approach to digital archiving has produced surprisingly affirmative responses:

UbuWeb posts much of its content without permission; we rip full-length CDs into sound files; we scan as many books as we can get our hands on; we post essays as fast as we can OCR them. And not once have we been issued a cease and desist order. Instead, we receive glowing e-mails from artists, publishers, and record labels finding their work on UbuWeb. (Goldsmith 2001)

In the mid-1990s, UbuWeb began with an emphasis on what was known variously as "concrete," "visual," or "shape poetry." Concrete poetry used the arrangement of words visually on the page or screen to create an effect and convey meaning. Another early UbuWeb interest included "found street poems," that is, "poems" in the form of street signs, advertisements, posters, and graffiti. With the emergence of streaming audio technologies, UbuWeb later expanded its ambit to include sound poetry — created and performed through a combination of literary and musical techniques. When MP3s became available and bandwidth limits increased, UbuWeb began adding video artworks to its collection, becoming an ever-increasing "repository for the "avant-garde" (Goldsmith 2011b). The archive now houses over 2,500 full-length streaming and downloadable films and videos. Additionally, in 2005, UbuWeb acquired "The 365 Days Project" - a collection of rare audio works, including tracks by the Russian-American conductor and composer Nicolas Slonimsky (1894-1995). The archive has broadened to include "ethnopoetic" audio works, including recordings of Inuit throat singing, as well as ethnopoetic visual poetry, such as native American song pictures. Ubu Editions is an open-access collection of PDFs on literature and poetry.

UbuWeb begins as a poetry community with the common intention of revitalizing concrete poetry in the public domain. The project exemplifies the notion of a web-based "gift economy" through radical approaches to distribution and access. Moreover, the term "nude media" describes the liberation of a digital file from its original context, including the UbuWeb platform itself, through peer-to-peer sharing systems (Goldsmith 2002, 4-5). Emancipated from the authority of the source or host, nude media create new networks of meaning. UbuWeb situates its archive of nude media squarely within a digital gift economy. In contrast to a commodity exchange or capitalistic model, a gift economy involves exchanges of goods, services, and intellectual property, with reciprocity (rather than money) expected. Whereas a commodity market ascribes power to those with the most capital, a gift-based market recognizes the act of giving as a value that ensures social cohesion and cultural longevity (Hyde 1999). Early anthropological research into gift economies focuses on the exchange networks of Native American and Canadian peoples. New media theorists have used the term to signify the capital-free economies that underpin knowledge-exchange in the information age (for an example, see Creative Commons licensing). The longevity of UbuWeb is, in part, made possible through an ethic of gifting its variable media acquisitions. For example, the Andy Warhol Audio Archive contains interviews with the artist from between 1965 and 1987, as well as audio recordings of contemporary Canadian filmmaker David 
Cronenberg reflecting on Warhol's evolution as an artist.

\section{Towards Archival Interoperability: Arts Preservation and Technology}

The previous section outlined the principal scoring systems for digital arts preservation, beginning with the Variable Media Questionnaire (VMQ) developed in the year 2000. Archival difficulties plague works of digital and media art. For instance, tape degradation will impact works of video art and failure to use archival quality paper and compromise the longevity of digital photographs. Bruce Wands describes three technological factors to consider in the preservation of digital artworks: data storage, bandwidth, and interactivity (Wands 2006, 33). In terms of the former factor, Mark Napier's net.flag demonstrates that many digital artworks are lost when user interactivity diminishes or access becomes constrained by technological obsolescence. Additionally, as the original media become degraded or simply outdated, the dual processes of documentation and preservation are ever more pressing. The truth is that forms of documentation can exist long after the work has been lost (Wands 2006, 33). Images of Internet artworks, critical commentaries about installations, or artist interviews about performative pieces are the strands of documentation coalesced by notation systems.

To a certain extent, the evolution of higher quality digital formats and increased bandwidth will continue to support data delivery, storage, and, ultimately, arts preservation. Moreover, technological development, including high-definition video and multichannel audio, will contribute to preservation efforts by increasing the capacity and efficiency of media. In addition, high-speed computer infrastructure - such as National Lambda Rail (NLR) in the US-will enhance the production and transmission of audiovisual works (Cisco Systems 2008). However, these technological strides alone will not ensure the future of the digital arts. In this respect, the continued refinement of notation systems is fundamental. Returning to the realities of "technological obsolescence," this section deepens our focus on key technologies and technical issues negotiated by digital arts conservators. Specifically, we explore the concept of interoperability as an approach used by digital archivists to ensure the long-term viability of artworks through extended collaboration between repositories and institutions. How is archival interoperability achieved through the technologies of metaservers, MPEG-21, and XML? Metaservers, the MPEG-21 metadata framework, and markup languages have been central to the interoperability of the VMQ, MANS, and PADS notation systems.

Firstly, the use of metaservers distributes digital arts preservation across different platforms. Building a more complete picture of the work, metaservers also heighten the accessibility of archival data, so information about digital artworks is not isolated from other networks or databases. Secondly, the MPEG-21 standard enhances multimedia access between artists and consumers across different networked platforms. Thirdly, markup languages, such as XML, are employed to establish relationships between the components of an artwork. These languages also support user participation while making possible the recording of the human and nonhuman components of a work so it can be re-created or re-performed (MacDonald 2009, 63). In this section, we conclude by returning to the new role of art museums and galleries in digital arts preservation. Indeed, digital arts practices have transformed museums into places of creation, documentation, technical innovation, and preservation with conservators and curators working cooperatively with artists, programmers, and technicians (MacDonald 2009, 62).

Interoperability is the state of effective and integrated information exchange. In addition to archival interoperability, there are other forms, including technical, semantic, political, community, legal, and international (Pelizzari 2012, 38). Interoperability realized through the use of a metaserver is a pivotal practice 
to the archival initiatives of the Variable Media Network (VMN).The second version of the Variable Media Questionnaire (VMQ2) offers partial flexibility, especially regarding the different components of a work that requires a unique, though interrelated, preservation plan. In contrast, the third version (VMQ3) (2010), designed by computer programmer John Bell, foregrounds flexibility by focusing on the relationships between parts of a work and manifests a higher level of interoperability. Bell's version is notable for its use of a metaserver, allowing communication about the artwork to transpire between different systems and platforms. By uploading data to the metaserver, the third iteration of the questionnaire links the VMQ architecture to external schemes, such as The Pool. Developed by Jon Ippolito, Joline Blais, and the Still Water Lab, "The Pool" is an online database for documenting the evolution of Internet artworks through different stages—-specifically "intent," "approach," and "release." "The Pool" includes descriptions and reviews of projects, as well as the relationships between projects in the database (Paul 2008, 174).

In order to foster interoperability, Bell deployed object-oriented programming (OOP) in which concepts are schematized as objects with attributes denoted in data fields. Additionally, VMQ3 makes use of open code programming, such as the open-source database management system MySQL and the programming languages PHP (Hypertext Preprocessor), HTML (HyperText Markup Language), CSS (Cascading Style Sheets), and JS (JavaScript) (Bell 2010). The metaserver searches for an artist's name and generates an identification list. It then returns a full record of all metadata related to the identification including titles of works, media, and links to different forms of documentation. In this way, a metaserver operates as a data repository tracing the relationships within and between artworks. The metaserver offers a place of convergence between online databases, such as "The Pool" and any other information system accessible via the API (Application Programming Interface). Thus, the aim of preserving digital artworks by carefully delineating their changes is enhanced through the principle of interoperability in which the documentation performed by dispersed institutions can be integrated.

In 1999, the concept of the MPEG-21 standard or the MPEG-21 Multimedia Framework emerges within the MPEG community in an effort to develop approaches to intellectual property management in digital environments (Cox, Tadic, \& Mulder 2006). Becoming a standard in 2003, MPEG-21 allows digital items to be declared and modified. Interoperability is one of the principal goals of the standard. MPEG-21 increases the exchange of multimedia resources among creators, users, and providers across an array of networked devices. The development of the MPEG-21, in part, signifies the blurring of the border line between private individuals (users) and production companies (creators). The standard makes intelligent use of metadata to establish an environment for content exchange. Different domains of MPEG-21 metadata include production details, descriptions of the interactions between users and the content, descriptions of content exchange and optimization, and guidelines specifying the legal use of the material (Cox et al., 2006, 48). The main concept behind the framework is the "digital item" (defined as any single multimedia file or a group of such files). The promotion of user interaction with the digital item is accomplished through direct distribution, modification, or consumption. The basis for transactions is a "digital item declaration," which defines and describes content using metadata about the owner, the media, and the parameters of its use according to copyright.

In addition to the MPEG-21 standard, markup languages, such as XML, are also essential to archival interoperability. Extensible Markup Language (XML) is used to exchange data across networks, create data sets for exchange across mismatched systems, and enable data translation from one format to another. XML simplifies the process of data exchange between two or more applications - a core function for platform 
interoperability (Kahate 2009, 5). For instance, the Media Arts Notation System (MANS) uses a form of XML known as Digital Item Declaration Language (DIDL). This language allows the creation of more detailed descriptions about digital objects. Specifically, DIDL enables the pronouncement of a digital item through the specification of its metadata and the relationships between its metadata and other artworks (Salomoni \& Mirri 2011, 28). The MANS produces a score of an artwork in three layers: the development of a conceptual model, the generation of a vocabulary to interpret the DIDL XML, and the production of a score as its top layer, resulting in a record of the artwork for future re-creation or re-performance.

Interoperable technologies have been indispensable to the digitization of artworks in library collections. While the technological landscape shifts perpetually and ephemeral artworks slip into disrepair or decline, libraries ensure digital heritage continuity between generations of users and artists (Bosma 2011, 177). Moreover, the archives of libraries act as repositories of data but, at the same time, offer content for subsequent creators (Bosma 2011, 178). The archiving of artworks at libraries and institutes using notation systems and other methods of preservation reminds us that a work of art is both "object and meme; it is an artist's work and a possible source of inspiration for other artists" (Bosma 2011, 178). Information theorist Prodromos Tsiavos recommends open-source frameworks for GLAMs, or galleries, libraries, archives, and museums (Tsiavos 2011). Commitment to an open-source ethos ensures interoperability between library archives and those of other cultural heritage institutions, such as museums and galleries. Founded in 2002 by curator Timothy Murray at Cornell University, the Rose Goldsen Archive of New Media Art is one of the first library-based archives of digital art. The archive preserves artworks based on CD, DVD, video, and the Internet, and includes a collection of catalogues, monographs, design catalogues, and other supporting materials.

Created by Janet Cohen, Keith Frank, and Jon Ippolito, “The Unreliable Archivist" (1998) is an example of an early Internet artwork that uses an established archive - äda'web - to critique the swiftly transforming role of the archivist in the digital era (see www.walkerart.org/collections/artworks/the-unreliable-archivist). Now preserved by the Walker Centre and originally designed for Netscape 4.07, the project begins as a response to the archiving of äda'web (1995-1998) by Benjamin Weil. "The Unreliable Archivist" interrogates the ethics of digital archiving through the use of a collection database-structured website that allows the user to disrupt or subvert the äda'web archive in unusual ways (Graham 2007, 102). For example, a user selects the style and layout of the website, and manipulates images taken from äda'web collection of new media art. The reconfiguration of the äda'web archive is executed according to the categories "language," "image," "style," and "layout." The user-determined metadata tags signal idiosyncratic departures from the usual archival categories: creator, title, date created, and medium. Some of the interactive features are just plain absurd (Graham 2010, 167-8). Texts, videos, and sound from äda'web are combined in unusual ways, allowing the user to "alter this archetypal äda'web page to suite [sic] your preferences" (Walker Art Center 9, 1998). Changing the category sliders gives the user the false appearance of choice.

Just how much should a collection change over time? What are the appropriate choices a user can make when participating in an artwork? What is the role of the conservator in the digital era? As a parody of the archival process, "The Unreliable Archivist" raises questions over how to preserve the dynamic and interactive qualities of many digital artworks. The work highlights some of the concerns of early new media archivists and queries the inherent limitations of archiving, particularly the condensing of complex projects into reductionistic metatags. Ippolito characterizes "The Unreliable Archivist" as "parasite art" that requires a host to feed on (Dietz 1999). The project maps metadata about äda'web, exploring the dynamics between a host and a parasite 
in digital domains, specifically in terms of archival structure and content. Indeed, the discourse of interactivity can be utopian and misplaced. Access occurs at physical and conceptual levels with metadata providing a window into the intellectual mechanisms of an artwork. The archivist's role as the mediator of interactivity, metadata, and the ultimate fate of the artwork is one not to be taken lightly.

\section{Works Cited}

Auslander, Philip. “The Performativity of Performance Documentation.” PAJ: A Journal of Performance and Art 28.3 (2006): $1-10$.

Barber, John. "Digital Archiving and 'The New Screen." Randy Adams, Steve Gibson, and Stefan Arisona, eds. Transdisciplinary Digital Art: Sound, Vision, and the New Screen. Berlin: Springer, 2008. 110-9.

Bell, John. “Archiving Experience: The Third Generation Variable Media Questionnaire.” V1.1 Retrieved, 26 July, 2013. $<$ http://thoughtmesh.net/publish/393.php>.

Bosma, Josephine. Nettitudes: Let's Talk Net Art. Rotterdam and Amsterdam: Nai Publishers and Institute of Network Cultures, 2011.

Brabham, Daren. Crowdsourcing. Cambridge: Massachusetts Institute of Technology, 2013a.

Brabham, Daren. "Crowdsourcing: A Model for Leveraging Online Communities." Aaron Delwiche and Jennifer Jacobs Henderson, eds. The Participatory Cultures Handbook. Abingdon: Taylor and Francis, 2013b. 120-9.

Cisco Systems. Case Study National LambdaRail Project. Casimer. DeCusatis, ed. Handbook of Fiber Optic Data Communication: A Practical Guide to Optical Networking 3 ed. Amsterdam: Elsevier, 2008. 399-402.

Claypoole Ted, and Payton Theresa. Protecting Your Internet Identity: Are You Naked Online? Plymouth: Rowman and Littlefield Publishers, 2012.

Cox Mike, Tadic Linda, and Mulder Ellen. Descriptive Metadata for Television: An End-to-End Introduction. Burlington: Elsevier, 2006.

Daniel Langlois Foundation. DOCAM Documentation Model, Retrieved, 25 July, 2013. $<$ http://www.docam.ca/en/Documentation -model.html>.

Dietz, Steve. "Archiving with Attitude: The Unreliable Archivist and ada'web." Retrieved 26 July, 2013. $<$ http://www.walkerart.org/gallery9/three/dietz_ua.html $>$.

Dietz, Steve. "Collecting New-Media Art: Just Like Anything Else, Only Different." Bruce Altshuler, ed. Collecting the New: Museums and Contemporary Art. Princeton: Princeton University Press, 2005. 85-102.

Fauconnier Ra, and Frommé Rens. "Capturing Unstable Media." Digital Culture and Heritage. 2004. Retrieved. $<$ http://citeseerx.ist. psu.edu/viewdoc/summary?doi=10.1.1.169.4766>.

Forging the Future. "Variable Media Questionnaire." Retrieved, July 17, 2013. <http://variablemediaquestionnaire.net/>.

Goldsmith, Kenneth. "UbuWeb Wants To Be Free.” July 18, 2013. <http://epc.buffalo.edu/authors/goldsmith/ubuweb. html>.

Goldsmith, Kenneth. "The Bride Stripped Bare: Nude Media and the Dematerialization of Tony Curtis." Retrieved, July 17, 2013. $<$ http://epc.buffalo.edu/authors/goldsmith/nude.pdf $>$.

Goldsmith, Kenneth. “Archiving Is the New Folk Art.” Retrieved, July 23, 2013. <http://www.poetryfoundation.org/harriet/2011/ 04/Archiving-is-the-new-folk-art/>.

Goldsmith, Kenneth. "UbuWeb." 2011b. <http://www.ubu.com/resources/index.html>.

Graham, Beryl. "Redefining Digital Art: Disrupting Borders." Fiona Cameron and Sarah Kenderdine, eds. Theorizing Digital Cultural Heritage: A Critical Discourse. Cambridge: The MIT Press, 2007. 93-112.

Graham, Beryl. "Tools, Methods, Practice, Process... and Curation." Hazel Gardiner and Charlie Gere, eds. Art Practice in a Digital Culture. Surrey: Ashgate, 2010. 165-74.

Gray, Stephen. "The Performance Art Documentation Structure." Retrieved, $25 \quad$ July, 2013. $<$ http://www.bristol.ac.uk/nrla/case-study/>.

Groys, Boris. Under Suspicion: A Phenomenology of Media. New York: Columbia University Press, 2012.

Hanhardt, John. "Introduction: The Challenge of Variable Media." Carey Ann Schaefer, Edward Weisberger, Yves Doucet, and Jacques Perron, eds. Permanence Through Change: The Variable Media Approach. New York: Guggenheim Museum Publications, 2003. 6-9. 
Harvey, Ross. Preserving Digital Materials. Berlin: Walter de Gruyter GmbH and Co., 2012.

Hyde, Lewis. The Gift: Imagination and the Erotic Life of Property. London: Vintage, 1999.

Internet Archive. "Welcome to the Prelinger Archives." Retrieved, 23 July, 2013. <http://archive.org/details/prelinger>.

Ippolito, John. "Accommodating the Unpredictable: The Variable Media Questionnaire.” Carey Ann Schaefer, Edward Weisberger, Yves Doucet, and Jacques Perron, eds. Permanence Through Change: The Variable Media Approach. New York: Guggenheim Museum Publications, 2003a. 47-53.

Ippolito, John. "Mark Napier, net.flag, 2002.” Francoise Charron, Trans. Carey Ann Schaefer, Edward Weisberger, Yves Doucet, and Jacques Perron, eds. Permanence Through Change: The Variable Media Approach. New York: Guggenheim Museum Publications, 2003b. 109-14.

Ippolito, John. "Learning from Mario: Crowdsourcing Preservation.” Retrieved, July 17, 2013.<http://three.org/ippolito/writing/ learning_from_mario/>.

Iseminger, Damian. "Works and Expressions in RDA: Problems and Solutions." Peter Lisius and Richard Griscom, eds. Directions in Music Cataloging. Middleton, WI: A-R Editions, Inc., 2012. 43-62.

Jones, Caitlin, and Muller, Lizzie. "Between Real and Ideal: Documenting Media Art.” Leonardo 41.4 (2008): 418-9.

Kahate, Atul. XML and Related Technologies. Delhi: Dorling Kindersley, 2009.

MacDonald, Corina. "Scoring the Work: Documenting Practice and Performance in Variable Media Art." Leonardo 42.1 (2009): 59-63.

Napier, Mark. “About net.flag.” Retrieved, 27 July, 2013. <http://webart.guggenheim.org/netflag/aboutF.html>.

Parikka Jussi, and Ernst Wolfgang. Digital Memory and the Archive. Minneapolis: University of Minnesota Press, 2013.

Paul, Christiane. "Digital Art/Public Art: Governance and Agency in the Networked Commons." Christa Sommerer, Lakhmi Jain, and Laurent Mignonneau, eds. The Art and Science of Interface and Interaction Design. Berlin: Springer, 2008. 163-85.

Pelizzari, Eugenio. "Harvesting for Disseminating: Open Archives and the Role of Academic Libraries." Audrey Fenner, ed. Managing Digital Resources in Libraries. New York: Routledge, 2012. 35-52.

Petrović-Šteger, Maja. "Spools, Loops, and Traces: On etoy Encapsulation and Three Portraits of Marilyn Strathern." Jeanette Edwards and Maja Petrović-Šteger, eds. Recasting Anthropological Knowledge: Inspiration and Social Science. Cambridge: Cambridge University Press, 2011. 145-64.

Prelinger, Rick. "Points of Origin: Discovering Ourselves through Access.” The Moving Image 9.2 (2009): 164-75.

Richmond, Alison. Conservation. Hoboken: Taylor and Francis, 2012.

Rinehart, Richard. "Appendices to a System of Formal Notation for Scoring Works of Digital and Variable Media Art." Archiving the Avant-Garde: Project Documents and Papers. 2004a. Retrieved. <http://www.bampfa.berkeley.edu/about/formalnotation apndx.pdf $>$.

Rinehart, Richard. "A System of Formal Notation for Scoring Works of Digital and Variable Media Art." Archiving the Avant-Garde: Documenting and Preserving Digital/Variable Media Art: Project Documents and Papers. 2004b. Retrieved. $<\mathrm{http} / / / w w w$. bampfa.berkeley.edu/about/formalnotation.pdf $>$.

Rinehart, Richard. "The Media Art Notation System: Documenting and Preserving Digital/Media Art." Leonardo 40.2 (2007): 181-7.

Salomoni Paola, and Mirri Silvia. “Adaptation Technologies in Mobile Learning.” Lee Chao, ed. Open Source Mobile Learning: Mobile Linux Applications. Hershey, PA: Information Science Reference, 2011. 18-34.

STARS. "Search: Richard Layzell.” Retrieved, 25 July, 2013. <http://stars.ilrt.bris.ac.uk/StarsWeb/stars?keywords=Richard\%20

Layzellandaction=search $>$.

Stringari, Carol. "Beyond 'Conservative': The Conservator's Role in Variable Media Preservation.” Carey Ann Schaefer, Edward Weisberger, Yves Doucet, and Jacques Perron, eds. Permanence Through Change: The Variable Media Approach. New York: Guggenheim Museum Publications, 2003. 54-59.

Tsiavos, Prodromos. "Extracting Value from Open Licensing Arrangements." Wolfgang Leister and Nils Christophersen, eds. Open Source, Open Collaboration and Innovation. Oslo: Norwegian Computing Center, 2011. 119-62.

UNESCO. Guidelines fo the Preservation of Digital Heritage. Paris, France: United Nations Education, Scientific, and Cultural Organization, 2003.

Van Saaze, Vivian. "Doing Artworks: An Ethnographic Account of the Acquisition and Conservation." No Ghost Just a Shell. Krisis: Journal for Contemporary Philosophy 1 (2009): 20-33. Retrieved. <http://www.krisis.eu/content/2009-1/2009-1-03saaze. pdf>. 
Walker Art Center 9. The Unreliable Archivist. Retrieved, 11 August, 2013. <www.walkerart.org/collections/artworks/theunreliable-archivist>.

Wands, Bruce. Art of the Digital Age. New York: Thames and Hudson, 2006.

Weinbren, Grahame. "Navigating the Ocean of Streams of Story." Retrieved, 22 July, 2013. $<$ http://www.guggenheim.org/images/content/pdf/new_york/ocean\%20of\%20streams.pdf $>$. 